\title{
Experiences in total knee arthtroplasty after distal femoral varus osteotomy
}

\author{
Bianca Röschke ${ }^{1}$, Wolfgang Hönle ${ }^{2}$, Alexander Schuh ${ }^{1 *}$ \\ ${ }^{1}$ Research Unit Orthopaedics and General Surgery, Neumarkt Clinic, Neumarkt, Germany; *Corresponding Author: \\ Alexander.Schuh@,klinikum.neumarkt.de; \\ ${ }^{2}$ Department of Orthopaedic Surgery, Neumarkt Clinic, Neumarkt, Germany.
}

Received 21 July 2010; revised 20 August 2010; accepted 25 August 2010.

\section{ABSTRACT}

Introduction: There is only little information available about total knee arthroplasty (TKA) following distal femoral varus osteotomy (DFVO). The aim of our study was to show our experiences and mid-term results of TKA after a previous DFVO. Material and method: In a retrospective study we identified 36 consecutive patients who had undergone TKA after a previous distal femoral varus osteotomy. The average duration of follow-up after the TKA was 8.2 years (min: 5.0, max: 9.2). X-rays were taken in 2 planes before TKA, 1 week after TKA and at latest follow-up. Tibiofemoral alignment was measured on weightbearing long-leg anteroposterior radiographs. Radiolucent lines at latest follow-up were documented. Functional evaluations were performed preoperatively and postoperatively (at the time of latest follow-up).

Results: The mean Knee Society knee score increased from 42 points before the arthroplasty to 91.3 points after the arthroplasty. The mean Knee Society function score increased from $\mathbf{2 7 . 4}$ points preoperatively to $\mathbf{9 3 . 2}$ points postoperatively. The mean overall Knee Society score increased from 91.3 points preoperatively to 163.4 points postoperatively. The mean radiographic alignment was $4.5^{\circ}$ of valgus $\left(10^{\circ}\right.$ of varus to $19^{\circ}$ of valgus) before TKA and $3.1^{\circ}$ of valgus (range, $3^{\circ}$ of varus to $6^{\circ}$ of valgus) at the time of latest follow-up. Postoperative complications included one deep vein thrombosis with non-lethal pulmonary embolism, one wound infection requiring revision and one septic loosening. Discussion: It is possible to perform TKA following DFVO with good mid-term results. In comparison to the literature there is no higher risk of complications in TKA following DFVO in comparison to primary TKA.

Keywords: Total Knee Arthroplasty; Distal Femoral Varus Osteotomy; Result; Complication; Knee Society Score

\section{INTRODUCTION}

Osteotomies around the knee for the purpose of unloading the affected compartment and correction of the underlying deformity are a well recognised treatment alternative for symptomatic osteoarthritis of the knee in active patients [1]. Varus deformity with medial compartment arthritis is more common than valgus deformity with lateral compartment involvement [1,2]. Several studies reported good outcomes lasting more than a decade for patients with varus deformity treated by high valgus tibial osteotomy in appropriately selected patients. For a valgus deformity of more than $12^{\circ}$ or in situations where the plane of the joint is deviated from the horizontal by more than $10^{\circ}$, a distal femoral varus osteotomy (DFVO) should be preferred [1]. A recent study indicated good mid-term success rates of DFVO even in the presence of moderate patellofemoral arthritis at a mean follow-up of 99 months [3]. Edgerton et al. [4] reported on 23 patients ( 24 knees managed with DFVO) and found $71 \%$ with good or excellent results at a mean follow-up of 8.3 years. In a recent study by Kosashvili et al. [1] 33 consecutive DFVOs (31 patients) with a minimum follow-up of ten years (mean 15.1, range 10-25) were reviewed. Fifteen DFVOs were converted to total knee arthroplasty (TKA) and one DFVO was awaiting TKA. The authors came to the conclusion that DFVO is reliably in delaying the need for TKA by more than a decade as conversion to TKA in less than ten years occurred in $6.1 \%$. Success of primary TKA with knee osteoarthritis is well established, and about $85 \%$ of patients are satisfied with the surgical outcome [5]. In the past, there have been reports of technical difficulties after 
failed high tibial head osteotomy that influenced outcomes of knee replacement. There is only little information available in TKA following DFVO [6-8]. The aim of our study was to show our experiences and mid-term results of TKA after a previous DFVO.

\section{MATERIAL AND METHODS}

In a retrospective study we identified 36 consecutive patients who had undergone TKA after a previous distal femoral varus osteotomy. The average duration of follow-up after the TKA was 8.2 years (min: 5.0 max: 9.2). The study group included 14 men and 22 women who had a mean age of 58.4 years (min: 27.8 , max: 81.5 ) at the time of the arthroplasty. 21 right knees and 14 left knees were involved. TKA was performed at an average of 9.2 years (min: 2.2, max: 35.8) after the osteotomies. The average height of the patients was $156.9 \mathrm{~cm}$ (min: 152, max: 178), the average weight was $78.2 \mathrm{~kg}$ (min: 51 , max: 108). All osteotomies had been internally fixed with a lateral blade-plate. Removal of the plates was preformed after 1-2 years after the osteotomy, at the time of TKA no hardware removal was necessary.

15 knees had osteoarthritis grade 3, 21 knees grade 4 according to Kellgren and Lawrence [9] preoperatively.

In all cases an intramedullary femoral alignment guide was used during TKA procedures. The operative notes did not indicate any modification of the starting hole in knees in which an intramedullary guide was used for alignment.

In all cases an intraoperative joint aspiration was performed, in one case an infection with Staph aureus could be detected, without any clinical signs for infection preoperatively.

Prostheses included 9 FS Knees (Protek, Switzerland, now Zimmer, Warsaw, Indiana) 2 Emotion Knees (Aesculap, Tuttlingen, Germany) and 25 PFC prostheses (DePuy, Johnson and Johnson, Warsaw, Indiana).

All procedures were performed in an ultra-clean-air theater (with antibiotic prophylaxis). During their stay at the hospital, all patients were treated with low molecular weight Heparin and compression stockings as a prophylaxis against deep vein thrombosis. For the duration of 6 weeks, partial weight bearing of $20 \mathrm{~kg}$ with the support of lower arm crutches was required.

$\mathrm{X}$ rays were taken in 2 planes before TKA, 1 week after TKA and at latest follow-up. Tibiofemoral alignment was measured on weightbearing long-leg anteroposterior radiographs. Radiolucent lines at latest follow-up were documented according to the Knee Society total knee arthroplasty roentgenographic evaluation system [10]. Functional evaluations were performed preoperatively and postoperatively (at the time of follow-up) with use of the 200-point system of the Knee Society [11]. The two components of this scale, the 100-point knee score and the 100-point function score, were extracted before and after the arthroplasty by means of a chart review performed after an average of 8.2 years of follow-up.

\section{RESULTS}

Mean operating time was 98.4 minutes (min: 55, max. 120).

Mean range of motion was Extension/Flexion 0/6/88 preoperatively and improved to Extension/Flexion 3/0/97 postoperatively.

The mean Knee Society knee score increased from 42 points (min: 15, max: 59) before the arthroplasty to 91.3 points (min: 85; max: 98) after the arthroplasty. The mean Knee Society function score increased from 27.4 points (min: 15, max: 60) preoperatively to 93.2 points (min: 50, max: 100 points) postoperatively. The mean overall Knee Society score increased from 91.3 points (min: 53, max: 127) preoperatively to 163.4 points (min: 110, max: 178) postoperatively.

$3 / 36$ cases had femoral notching intraoperatively. In these cases partial weight bearing was requested for 10 weeks, further follow-up was uneventfull in all 3 cases. Postoperative complications included one deep vein thrombosis with non-lethal pulmonary embolism, one wound infection requiring revision and one septic loosening requiring 2 stage revision arthroplasty.

The mean radiographic alignment was $4.5^{\circ}$ of valgus $\left(10^{\circ}\right.$ of varus to $19^{\circ}$ of valgus) before TKA and $3.1^{\circ}$ of valgus (range, $3^{\circ}$ of varus to $6^{\circ}$ of valgus) at the time of latest follow-up. At latest follow-up mean femoral angle $\alpha$ was $97.1^{\circ}$ (min: 88, max: 104). Mean tibial angle $\beta$ was $98.1^{\circ}$ (min: 85, $\left.\max : 95\right)$. Mean femoral flexion angle $\gamma$ was $3.9^{\circ}$ (min: 1.2 , max: 8). Mean tibial angle $\delta$ was $89^{\circ}$ (min: -3 , max: 9).

Radiolucent lines according to the Knee Society total knee arthroplasty roentgenographic evaluation system are shown in Table 1.

\section{DISCUSSION}

Distal femoral varus osteotomy (DFVO) is indicated for patients with isolated lateral compartment osteoarthritis of the knee with associated valgus deformity of the knee. Aim of this procedure is delaying TKA. But DFVO can only be recommended if the results of TKA following DFVO are comparable to primary TKA and if there are not more complications. The ideal patient has isolated lateral compartment arthritis with a moderate valgus deformity, is physiologically young, has an occupation or activity level that makes arthroplasty less appropriate, and has a normal body-mass index and satisfactory range of motion and stability of the knee $[8,12]$. 
Table 1. Radiolucent lines according to the Knee Society Total Knee Arthroplasty Roentgenographic Evaluation and Scoring System (4).

\begin{tabular}{cccccccc}
\hline \multicolumn{7}{c}{ AP view of the tibial component } \\
\hline Zone & 1 & 2 & 3 & 4 & 5 & 6 & 7 \\
Count & 10 & 4 & - & 5 & - & - & - \\
\hline \multicolumn{7}{c}{ Lateral view of the tibial component } \\
\hline Zone & 1 & 2 & 3 & & \\
Count & 5 & - & - & & \\
\hline & Lateral view of the femoral component \\
\hline Zone & 1 & 2 & 3 & 4 & 5 & 6 & 7 \\
Count & 1 & - & 1 & 1 & - & - & - \\
\hline
\end{tabular}

Several studies reported good outcomes lasting more than a decade for patients with varus deformity treated by high valgus tibial osteotomy in appropriately selected patients. Instead, for a valgus deformity of more than $12^{\circ}$ or in situations where the plane of the joint is deviated from the horizontal by more than $10^{\circ}$, a DFVO should be preferred. DFVO is reliably in delaying the need for TKA by more than a decade as conversion to TKA in less than ten years occurred in $6.1 \%$ in a recent study (8). Several studies have investigated the effects of proximal tibial osteotomy on the results of subsequent TKA $[2,13,14]$. Some studies have demonstrated increased difficulties and higher complication rates when the results of conversion of a previously osteotomized knee to a TKA are compared with those of primary TKA. Specific difficulties have included more difficult exposure secondary to patella infera and wound-healing difficulties, leading to increased risks of patellar tendon avulsion and infection, respectively.

Only few studies have evaluated the effects of DFVO on the results of subsequent TKA [6-8].

We can follow Nelson et al. [8] that the resulting deformity following DFVO is extraarticular; therefore, intra-articular correction during TKA may lead to ligamentous instability that in some cases is not correctable with ligament releases. In our series we had no difficulties with collateral ligament balancing. In all cases a non-constrained prosthesis could be used. The extraarticular varus deformity of the femur following DFVO often results in a situation in which the femoral anatomical axis intersects the lateral femoral condyle rather than the intercondylar notch. Therefore, when intramedullary alignment is used, the starting hole should be placed where the femoral anatomic axis intersects the distal part of the femur at the knee. A possibility to solve this problem is a careful preoperative templating and determination of the appropriate location of the starting hole. Another option is to use an extramedullary femoral alignment or a computer assisted surgery navigating device. Maybe femoral notching can be minimized or better avoided by using a navigation system.

On the basis of their results, Nelson et al. [8] concluded that TKA decreases pain and improves knee function in patients who have had a previous DFVO with subsequent development of instability and/or end-stage posttraumatic arthritis. The malposition rate was relatively high (7/11), and the results were not as good. The mean Knee Society knee score was 35 points before the arthroplasty and 84 points after the arthroplasty. The mean Knee Society function score was 49 points before the arthroplasty and 68 points after the arthroplasty. The mean interval between the femoral osteotomy and TKA was fourteen years (min: 2, max: 32). A constrained prosthesis was required in five of the eleven knees. Two knees had an excellent result, five had a good result, and four had a fair result.

In our series, which is the largest series ever published, the mean Knee Society knee score increased from 42 points (min: 15, max: 59) before the arthroplasty to 91.3 points (min: 85; max: 98) after the arthroplasty. The mean Knee Society function score increased from 27.4 points (min: 15, max: 60) preoperatively to 93.2 points (min: 50, max: 100 points) postoperatively. The mean overall Knee Society score increased from 91.3 points (min: 53, max: 127) preoperatively to 163.4 points (min: 110, max: 178) postoperatively. Whereas we achieved better clinical results in comparison to Nelson et al. [8], we achieved comparable radiologic results. In the series of Nelson et al. [8] the mean radiographic alignment was $3.6^{\circ}$ of valgus (range, $7^{\circ}$ of varus to $18^{\circ}$ of valgus) before the arthroplasty and $3.3^{\circ}$ of valgus (range, $1^{\circ}$ of valgus to $6^{\circ}$ of valgus) at the time of the latest follow-up.

In one case we could detect Staph. aureus intraoperatively. In this case 2 stage revision arthroplasty was necessary due to deep infection of the TKA. We therefore recommend that all patients who receive a TKA following a DFVO should have preoperative joint aspiration as well as intraoperative gram-staining and frozen-section analysis to minimize infection rate.

In comparison to the literature there is no higher risk of complications in TKA following DFVO in comparison to primary TKA [5].

In a small series of eight cases of TKR following supracondylar varus femoral osteotomy Cameron et al. [7] found that the distal femur was offset medially on the femoral diaphysis; the outcome as measured by the clinical result and the alignment was uniformly excellent. Cameron et al. [7] concluded, that supracondylar varus femoral osteotomy did not affect the subsequent TKR. 
In our study TKA was performed at an average of 9.2 years (min: 2.2, max: 35.8) after DFVO. The average duration of follow-up after TKA was 8.2 years. According to our results we postulate that it is possible to perform TKA following DFVO with good mid-term results. We could not find any technical difficulties in TKA following DFVO. Therefore we can recommend DFVO as a reliable procedure in delaying the need for TKA in patients with osteoarthritis of the lateral compartment with a valgus deformity.

\section{REFERENCES}

[1] Kosashvili, Y., Safir, O., Gross, A., Morag, G., Lakstein, D. and Backstein, D. (2010) Distal femoral varus osteotomy for lateral osteoarthritis of the knee: A minimum ten-year follow-up. International Orthopaedics, 34, 249254.

[2] van Raaij, T.M., Reijman, M., Furlan, A.D. and Verhaar, J.A. (2009) Total knee arthroplasty after high tibial osteotomy. A systematic review. BMC Musculoskelet Disord, $20(\mathbf{1 0}), 88$.

[3] Wang, J.W. and Hsu, C.C. (2006) Distal femoral varus osteotomy for osteoarthritis of the knee. Surgical technique". The Journal of Bone and Joint Surgery, 88(Supp I), 100-108.

[4] Edgerton, B.C., Mariani, E.M. and Morrey, B.F. (1993) Distal femoral varus osteotomy for painful genu valgum. A five to 11 year follow up study. Clinical Orthopaedics and Related Research, 288, 263-269.

[5] National Institutes of Health (2003) NIH consensus statement on total knee replacement. NIH Consensus and
State-of-the-Science Statements, 20, 1-34.

[6] Beyer, C.A., Lewallen, D.G. and Hanssen, A.D. (1994) Total knee arthroplasty following prior osteotomy of the distal femur. American Journal of Knee Surgery, 7, 2530.

[7] Cameron, H.U. and Park, Y.S. (1997) Total knee replacement after supracondylar femoral osteotomy. American Journal of Knee Surgery, 10(2), 70-71.

[8] Nelson, C.L., Saleh, K.J., Kassim, R.A., Windsor, R., Haas, S., Laskin, R. and Sculco, T. (2003) Total knee arthroplasty after varus osteotomy of the distal part of the femur. The Journal of Bone and Joint Surgery, 85(6), 1062-1065.

[9] Kellgren, J.H. and Lawrence, J.S. (1957) Radiological assessment of osteoarthrosis. Annals of the Rheumatic Diseases, 16(4), 494-502.

[10] Ewald, F.C. (1989) The Knee Society total knee arthroplasty roentgenographic evaluation and scoring system. Clinical Orthopaedics and Related Research, 248, 9-12.

[11] Insall, J.N., Dorr, L.D., Scott, R.D. and Scott, W.N. (1989) Rationale of the knee dociety clinical rating system. Clinical Orthopaedics and Related Research, 248, 13-14.

[12] Pach, M., Uvízl, M., Holibka, R. and Zapletalová, J. (2005) Varus supracondylar osteotomy of the femur-longterm results. Acta Chirurgiae Orthopaedicae et Traumatologiae Cechoslovaca, 72, 363-370.

[13] Katz, M.M., Hungerford, D.S., Krackow, K.A. and Lennox, D.W. (1987) Results of total knee arthroplasty after failed proximal tibial osteotomy for osteoarthritis. The Journal of Bone and Joint Surgery, 69(2), 225-233.

[14] Windsor, R.E., Insall, J.N. and Vince, K.G. (1988) Technical considerations of total knee arthroplasty after proximal tibial osteotomy. Journal of Bone and Joint Surgery, 70(4), 547-555. 